\section{Fluid flow enhances vascularization and maturation of kidney organoids}

Current protocols for the development of kidney organoids generate 3D aggregates containing nephron components. However, vascular development within these organoids is limited, and researchers have relied on animal transplantation to generate perfusable vasculature. A new paper demonstrates that culturing kidney organoids under conditions of flow enhances vascularization and the maturation of nephron components. "Glomerular vascularization is particularly critical for generating blood filtration structures that are necessary for urine production, and we suspected that the lack of vascular incorporation into glomeruli and consequential lack of multilineage crosstalk might be one reason why kidney organoids do not contain morphologically mature podocytes," say Ryuji Morizane and Navin Gupta. "We show that simply flowing fluid across the surface of kidney organoids on an adherent extracellular matrix dramatically enhances both their vascularization and maturation in vitro without the need for animal implantation," add Kimberly Homan and Jennifer Lewis.

To apply mechanical flow to kidney organoids, the researchers placed partially differentiated kidney organoids onto an adherent layer of extracellular matrix housed within a $3 \mathrm{D}$ printed millifluidic chip. Application of flow over the organoid surface led to enhanced differentiation and expansion of the endogenous endothelial cell population. "As a result, the kidney organoids form robust vascular networks that can anastomose between adjacent organoids," explain Homan and Lewis. "Their epithelial and glomerular regions also polarize and mature to a far greater extent than organoids subjected to static culture conditions, showing that $3 \mathrm{D}$ vascularized

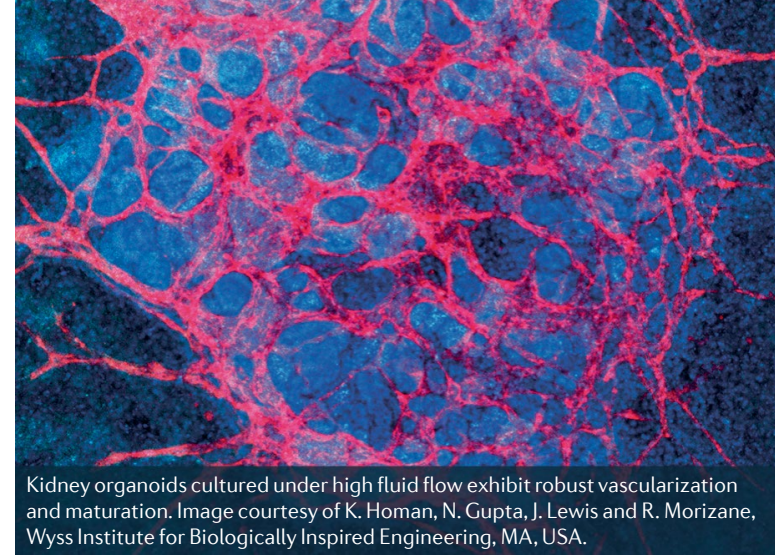

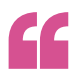

Simply flowing
fluid across
the surface
of kidney
organoids ...
dramatically
enhances
both their
vascularization
and maturation

organoid platforms better recapitulate the architecture and function of in vivo organ-specific tissues than static cultures." Moreover, they found that maintenance of intrinsic vascular endothelial growth factor signals was crucial for the development of vascularized nephron structures.

The researchers believe their vascularized kidney organoids provide a new platform for drug testing and disease modelling. "Our ultimate goal is to design and construct kidney organoid-based devices that enable filtration and reabsorption in vitro," they say.

Susan J. Allison

ORIGINAL ARTICLE Homan, K. A. et al.

Flow-enhanced vascularization and maturation of kidney organoids in vitro. Nat. Methods https://doi.org/10.1038/s41592-019-0325-y

\title{
FIBROSIS
}

\section{Ubiquitylation of PTEN drives fibrosis in diabetic kidney disease}

Diabetic kidney disease (DKD) is a serious complication of diabetes mellitus, with progressive renal fibrosis often leading to end-stage renal disease. Factors such as hyperglycaemia, growth factors and the transcription factors TWIST and SNAI1 are known to contribute to fibrosis, but approaches to therapeutically target these factors are limited. New findings show that a modified form of phosphatase and tensin homologue (PTEN) - characterized by linkage of a polyubiquitin chain at lysine 27 (referred to as PTEN ${ }^{\text {K27-polyub }}$ — promotes partial epithelial-to-mesenchymal transition (EMT) and renal fibrosis in DKD and can be targeted to improve renal outcomes

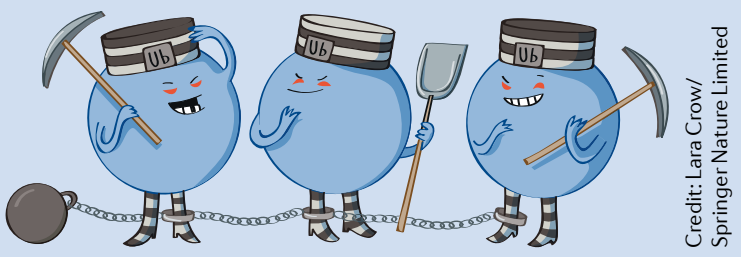

in models of DKD. "Our studies demonstrate that PTEN ${ }^{\text {K27-polyub }}$ can be used for the early diagnosis of DKD and that targeting PTEN ${ }^{\text {227-polyUb }}$ with the small molecule triptolide is a new therapeutic option for kidney fibrosis," say Liuqing Yang and Chunru Lin.

Yang and Lin say that their previous work, showing that PTEN ${ }^{\mathrm{K} 27-\text { polyUb }}$ correlates with EMT in renal tubular epithelial cells, led them to propose that PTEN ${ }^{\text {K27-polyub }}$ could be used to stratify patients at risk of DKD. PTEN ${ }^{\text {K27-polyUb }}$ promotes dephosphorylation and stabilization of TWIST and SNAI1, resulting in enhanced EMT. In mice, genetic inhibition of PTEN ${ }^{\text {227-polyUb }}$ attenuated histological and functional indices of kidney injury induced by Col4a 3 deletion, or by administration of folic acid or streptozotocin. In patients with diabetes mellitus, serum and urine levels of PTEN ${ }^{\text {K27-polyUb }}$ were higher than those of healthy controls and correlated negatively with estimated glomerular filtration rate.

The researchers then used a high-throughput assay to screen small molecular inhibitors that target MEX3C - the E3 ligase that catalyses

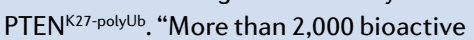
compounds were tested using this method and triptolide was identified as one of the top candidates that effectively inhibited MEX3C enzymatic activity," say Yang and Lin. Treatment with triptolide inhibited PTEN ${ }^{\mathrm{K} 27-\text { polyUb }}$ mediated EMT of renal epithelial cells, reduced levels of TWIST and SNAI1 and improved outcomes in models of DKD.

The researchers say their study findings suggest that identification of PTEN ${ }^{\text {K27-polyUb }}$ might facilitate early diagnosis of DKD and represent a viable therapeutic target. "We plan to validate PTEN ${ }^{\text {27-polyUb }}$ as a biomarker in a larger cohort of patients in order to further demonstrate its value in stratifying at-risk patient populations," they say.

Susan J. Allison

ORIGINAL ARTICLE Li, Y. et al. PTEN-induced partial epithelial-mesenchymal transition drives diabetic kidney disease. J. Clin. Invest. https://doi. org/10.1172/JCl121987 (2019) 